\title{
Effect of jar shape on high-energy planetary ball milling efficiency: Simulations and experiments
}

\author{
M. Broseghini ${ }^{a}$, M. D'Incau ${ }^{a,{ }^{*}}$, L. Gelisio ${ }^{a}$, N.M. Pugno ${ }^{b, c, d}$, P. Scardia \\ a Department of Civil, Environmental \& Mechanical Engineering, University of Trento, via Mesiano, 77, 38123 Trento, \\ Italy \\ ${ }^{b}$ Department of Civil, Environmental \& Mechanical Engineering, Laboratory of Bio-Inspired and Graphene \\ Nanomechanics, University of Trento, via Mesiano, 77, 38123 Trento, Italy \\ c Center for Materials and Microsystems, Fondazione Bruno Kessler, Via Sommarive 18, 38123 Povo (Trento), Italy \\ a School of Engineering and Materials Science, Queen Mary University of London, Mile End Road, London E1 4NS, \\ United Kingdom
}

*Corresponding author. E-mail address: mirco.dincau@unitn.it.

\begin{abstract}
Enhanced comminution in a planetary ball mill was achieved by suitably re-designing the jar shape. Compared with a traditional cylindrical vial of circular cross-section, the new jar was modified internally to have a flat wall portion resulting in a half moon cross-section. Results from simulations using a multibody dynamics software, suggest that this geometry increases the number of highvelocity collisions with energy exchange along the axial direction, deemed as more effective in the comminution process. X-ray diffraction line profiles of calcium fluoride (CaF2) ground in the two jars under equivalent conditions were used to obtain information on the microstructure resulting from the milling process and validate the modelling results. A better homogeneity and a faster reduction of crystallite size were achieved using the new design compared to that using the standard cylindrical vial design. Optimal operating conditions, in terms of jar-to-plate angular velocity ratio, are correlated and discussed according to the model predictions.
\end{abstract}

\section{Keywords}

Ball-milling, Jar shape, Numerical modeling, X-Ray Diffraction

\section{Introduction}

Planetary ball milling is a widespread and versatile technique for the structural and microstructural properties tuning of almost any kind of material. It is often used to grind materials down to the nanoscale, generally providing both comminution and incorporation of defects by extensive plastic deformation, thus supplying the conditions for mechanical activation [1,2]. Remarkable examples are the mechanical alloying of metals as well as the direct and indirect synthesis of ceramics [3-5]. The method has also proved to be effective in the mechanical exfoliation of bulk systems, for the largescale production of two-dimensional nanostructured materials - such as graphene and boron nitride nanosheets [6,7], nowadays of utmost scientific and technological interest. Although rather simple in terms of geometry and working principles (see Fig. 1 and e.g. [8]), the versatility and efficiency of the planetary ball mill depend very much on the appropriate tuning of a multitude of milling variables. Among the others, the number and size of the balls, the jar geometry and the velocity of revolving parts should certainly be mentioned. The setup parameters strongly influence the ball trajectories, which, in turn, determine the nature and magnitude of the impulsive forces transferred by milling media collisions. These forces can be divided into normal and shear components: the former should promote fracture while shear components induce plastic deformation $[9,10,6]$. 
As an alternative to extensive experimental testing, deemed as expensive and time-consuming to the point of being most often unfeasible, a dynamic-mechanical model of planetary ball milling was adopted [11] to fine-tune milling parameters and establish correlations with the end-product. This model, based on a simple contact scheme depending on a few easily estimable parameters, supplies quick and accurate predictions of system efficiency under different operating conditions and is, in principle, valid for any material of interest. The effect of jar $(\omega)$ and plate $(\Omega)$ velocities was investigated, providing a detailed picture of the kinematics and dynamics of the milling bodies, together with a description of contact events, supporting an outright understanding of ball motion regimes. An assessment of the kinetic energy available, both along normal and tangential directions, was obtained and results were validated against experimental data. In particular, best milling conditions, corresponding to the highest impact energy, were found to correlate with the most disordered ball motion, which develops within a well-defined range of jar-to-plate velocity ratios $(\omega /$ $\Omega+1)$.

Beyond the influence of plate and jar angular velocities, the present work investigates the effect of jar shape on the process efficiency. Particularly, the ratio of normal-to-shear transferred loads was considered to be an essential parameter in the design of end product characteristics, in terms of structure and microstructure. To increase the number of collisions with high-normal-velocity component, a new type of jar was designed. Simulation results, obtained using the above-mentioned multibody dynamics model, were analyzed in terms of kinetic energy, balls trajectories and probability distribution of velocities, in both the normal and tangential directions. The experimental validation was supported by X-ray Powder Diffraction Line Profile Analysis (XRPD-LPA) of ground calcium fluoride (CaF2).

\section{Methods}

Planetary ball mills consist of two or more jars rotating both around its own axis and the line of symmetry of the supporting plate (radius $R P=125 \mathrm{~mm}$, see Fig. 1). The motion of the milling media (balls) inside the vial, which is driven by the resulting field of two centrifugal forces and the Coriolis force, causes impacts that transfer compressive and shear forces to the powder charge. These forces lead to structural and microstructural alterations and/or mechanochemical effects $[13,14]$.

As shown by Burgio et al. [15], at optimum milling conditions the trajectory ideal for a single ball follows a point on the vial circumference until the resulting field of forces acts to carry the ball across the jar to the opposite point, perpendicularly to the jar surface. In a real scenario, trajectories depend on the interactions between many balls and forces are exchanged in every possible direction with respect to the impact reference frame. To enhance the normal component of the transferred force, a half-moon shaped jar (HM) is proposed, with a design based on a flat surface halfway between the original curved wall and the vial axis. This way, when balls rolling along jar wall approach the flat surface, they are forced to follow the ideal path, therefore increasing the amount of energy along impact axis and thus causing a more intense comminution effect.

\subsection{Computer simulations}

Computer simulations are a useful tool to explore thoroughly and characterize the milling process, providing insights into kinematic and dynamic quantities, mostly inaccessible to experiments. In this study, the Fritsch Pulverisette 4 (P4 [16]) planetary ball mill, equipped with either the Cylindrical (CY) or the HM jar, was modelled using the multibody dynamics software MSC.Adams [17], as reported in [11]. Twelve steel balls (radius $6 \mathrm{~mm}$ ) were placed randomly inside each jar and angular velocities $\omega$ and $\Omega$ were applied to the two cylindrical shafts connecting the revolving parts (physical properties of the milling media and jars are reported in Table 1). Owing to the intrinsic multibody nature of the system, the solution of the Lagrangian equations of motion of the milling media was determined using the Hilbert-Hughes-Taylor (HHT, $[18,19])$ integrator, with automatic step tuning and maximum numerical error of 10-8. Simulations lasted $24 \mathrm{~s}$ but, to allow motion homogenization after media 
insertion, the first $4 \mathrm{~s}$ were discarded during data analysis. It is worth noting that the powder charge, typically a few percent volume of the milling media, was not explicitly modelled but accounted for by a suitable selection of contact and friction parameters. This choice was based on the work by Rosenkranz et al. [20] who, by inspecting camera images revealing the formation of powder coatings on balls and jar surfaces, concluded that the presence of the mill feed strongly affects friction coefficients while not substantially influences restitution and therefore damping properties. To account for friction within the MSC.Adams model, the force prescribed by the (hard-coded) Coulomb model was added to the equation of motion with the friction coefficient, expressed as a function of slip velocity, smoothly varying from the static $(\mu \mathrm{s})$ to the dynamic $(\mu \mathrm{d})$ value. These parameters can be experimentally determined, for example by pin-on-disc tribological tests [21], for each couple of analyzed materials.

The contact scheme plays a crucial role, as it rules the interactions among rigid bodies. The hardcoded MSC.Adams impact function, belonging to the continuous approach [22], adds a forcedisplacement law to the equation of motion, including the contribution of a non-linear spring, Fk, and a linear damper, Fd, (Dubowsky and Freudenstein impact pair model [23]),

$$
F_{c}=F_{k}+F_{d}=k u^{n}+c \dot{u},
$$

where $u$ and $u_{\text {_ }}$ are the relative displacement and velocity of colliding bodies, whereas $\mathrm{k}$ and $\mathrm{c}$ are the spring generalized stiffness and the damping coefficient, respectively. To prevent discontinuities, $\mathrm{c}$ varies from 0 to a maximum value, cmax, depending on the relative displacement of colliding bodies. An estimate of cmax was determined from the classical solution of the damped vibration of a mass spring system [23], starting from the experimental measurement of the coefficient of restitution (ball-against-plane drop test [24]), e. Then, a parametric numerical analysis provided the $u$ value at which cmax has to be applied. For the spring component, fixing $n=3 / 2$, an estimate of $k$ was derived from the Hertzian theory of contact [25], applied both to the ball-ball and to the ballplane (approximating the jar surface) impacts. Values of parameters introduced in contact and friction schemes in this study, are reported in Table 2.

The typical simulation output consists of a complete set of dynamic variables for milling media as well as quantities pertaining to each contact event, thus allowing for a full understanding of the evolution of the investigated system. Moreover, each computed quantity can be decomposed into normal and tangential components with respect to the local impact reference frame, thus highlighting preferential directions for energy exchange between milling media and mill feed. The most intuitive quantities to investigate are the trajectories of milling media, offering a quick understanding of the complexity of the motion, directly related to the energy transfer mechanism (see [11]). To compare the efficiency of the different jars, it is also useful to calculate the impact kinetic energy of the system $(\mathrm{El})$, normalized over jar volume (V) and simulation time ( $\mathrm{T})$, i.e. the impact power density. This quantity is computed in the impact reference frame to provide an upper bound estimate of the energy available for grinding the mill charge [26-29,20],

$$
\frac{E_{l}}{(\tau V)}=\frac{1}{2 \tau V} \sum_{j=1}^{C} \frac{m_{1 j} m_{2 j}}{m_{1 j}+m_{2 j}} \dot{u}_{j}^{2},
$$

where $\mathrm{mi}$ is the mass of the $\mathrm{i}$-th colliding body (the mass of the jar is assumed to be infinity) and $\mathrm{u}_{-}$ the relative velocity. The summation runs over $\mathrm{C}$, which is the number of points sampling collisions during the simulation time period $(\mathrm{T})$ with mean output step size of $\sim 3 \mathrm{E}-3$ (integrator parameters are hmax $=1 \mathrm{E}-6$ and step = 100). It must thus be stressed that the absolute scale for the power impact density implicitly depends on the choice of these parameters and therefore is arbitrary and not directly comparable with power available in a real apparatus. The normalization factor $\mathrm{V}$ accounts for the different internal volume of the jars, respectively $72 \mathrm{ml}(\mathrm{CY})$ and $62 \mathrm{ml}(\mathrm{HM})$, limiting the space for ball motion and thus the transferred energy. By introducing the components of the relative velocity 
along $\left(u_{-} n\right)$ or perpendicular $\left(u_{-} t\right)$ to the impact axis, this quantity can be decomposed into its normal and tangential components, thus highlighting the preferential mode of energy exchange. Finally, an additional view of the energy exchange mechanism, completing the comparison between $\mathrm{CY}$ and $\mathrm{HM}$ jars, is provided by the probability distribution of the relative velocities of colliding bodies during impacts.

\subsection{Experimental techniques}

X-Ray powder diffraction (XRPD) was employed to characterize the structure and microstructure of the end product, in order to assess the effect of grinding conditions and validate the model. XRPD patterns were collected using CuKa radiation and a Rigaku PMG/VH diffractometer with a set of optical components (slits and bent graphite analyzer crystal) producing a narrow and symmetrical instrumental line profile across a wide angular region. The instrumental response was assessed by measuring a NIST SRM sample (660aLaB6, [30]), modelled by pseudoVoigt functions and parameterized to be used in following analyses of the powder pattern [31]. Ball milled fluorite samples were analyzed under the same experimental conditions and details can be found in $[11,32]$. The powder patterns were analyzed by Whole Powder Pattern Modelling (WPPM, [31-34]), assuming a lognormal distribution of spherical nanocrystalline domains and dislocations generated by the grinding process [35,37]. Besides information on mean size $(\langle D\rangle)$ and dispersion of the scattering domains, the WPPM analysis provides data on the inhomogeneous strain distribution, which can be related to the dislocation density $(\rho)$ according to the theory of Krivolgalz and Wilkens (see [31] and references therein). As an example of WPPM analysis, XRPD experimental data and modelling results for pristine and ground fluorite ( $\mathrm{HM}$ jar, $\omega / \Omega+1=-2.2)$ are reported in Fig. 2 .

\section{Results and discussion}

The main purpose of this work was to investigate the different grinding actions of the CY and HM jars and the dependence of the process ef- ficiency on the plate $(\Omega)$ and jar $(\omega)$ velocities. To properly account for mill charge and to compare numerical and experimental data collected on ground fluorite samples, two extreme conditions were simulated, namely the (i) steel-steel (i.e. milling media only, no mill charge) and the (ii) steel-fluorite interactions (i.e. jar surface completely covered by fluorite). The experimental case was expected to fall between these two boundary conditions. As previously discussed, no parameters were changed apart from the friction coefficients (Table 2) to simulate the two cases.

Fig. 3 illustrates the impact power density (Eq. (2)) for steel-steel interaction for sets of simulations performed at different fixed angular plate velocities $\Omega$ and increasing $\omega$. Interestingly, $\Omega$ strongly affects the amount of energy exchanged in the process, whereas it moderately alters the location, in terms of $\omega / \Omega+1$, of the maximum of the curve, corresponding to the maximum overall efficiency of the process. While the CY jar transfers the largest power at approximately $\omega / \Omega+1=-2.6$, the optimal condition of the $\mathrm{HM}$ jar, although less energetic than its $\mathrm{CY}$ counterpart, extends over a wider range, from $\omega / \Omega+1=-1.8$ to -2.2 . The HM jar, therefore, has a more robust grinding action, being less sensitive to variations in milling parameters. Moreover, it should be noted that the volume and the longest free path for ball motion is smaller for the HM than for the $\mathrm{CY}$ jar, thus resulting in reduced acceleration, velocity and kinetic energy transfer from the milling media to the powder. The analysis of ball trajectories reveals a correlation between motion regimes and energetic efficiency (see Fig. 3). In particular, by decreasing the angular velocity ratio, both jars demonstrate an increasingly disordered cascading motion, until it turns into a cataracting regime, providing the best milling conditions (see [11]). Above a threshold value of angular velocity ratio, balls tend to roll on (CY) or stitch to $(\mathrm{HM})$ the jar surface, therefore strongly limiting the amount of transferred energy.

Fig. 4 reports the ball-ball (a) and ball-jar (b) components of the impact power density calculated for $\Omega=200 \mathrm{rpm}$, further divided into normal and tangential components with respect to the local impact 
reference frame. In both cases, contacts among balls clearly play the major role and the largest power is exchanged in the tangential direction. However, it is interesting to notice that the ratio of energy transferred in the normal direction to the total energy (El(normal/total)) is larger for the HM than for the $\mathrm{CY}$ jar: this information is crucial to design a milling process exchanging energy along a specific direction.

The probability distribution of the relative velocities of colliding bodies is reported in Fig. 5 for both jars. Even if the overall efficiency, as already pointed out, is higher for CY than for HM jar, the comparison of relative velocities offers insights into the typical ranges of exchanged energies. Before and at the angular velocity ratio $\omega / \Omega+1=-1.8$, corresponding to the $\mathrm{HM}$ jar optimal milling conditions (upper part of Fig. 5), for the tangential component the $\mathrm{CY}$ jar exhibits a larger number of highrelative-velocity events (say, $\mathrm{N} 500 \mathrm{~mm} / \mathrm{s}$ ). Turning to the normal velocity, by contrast, the HM jar performs better. Although the relative frequency of high-velocity impacts is tiny, it should be noticed that this type of impact exchanges the highest amount of energy, and is, therefore, the most effective in the comminution process. In fact, fragmentation and related phenomena (e.g. work-hardening) are strictly nonlinear, so the sum of energy released by low-relative-velocity contacts may not be as significant as that associated to a single high-relative-velocity event [38]. In particular, impacts along the normal direction are supposed to fracture brittle powders more efficiently than impacts with a strong tangential component $[9,10,6]$; therefore, as the fraction of significant events along the normal direction is higher in $\mathrm{HM}$ than in the $\mathrm{CY}$ jar, the former is expected to be more effective in size reduction of brittle materials such as fluorite.

Fig. 6 depicts the map of the balls-jar contact events and their magnitude in terms of relative velocity in normal direction for $\omega / \Omega+1=-1.8$. While in the $C Y$ jar the collision density is uniform, this is not true for the HM jar, where regions with higher impact density/magnitude exist, particularly concentrated near the upper corner. In agreement with $\omega$ and $\Omega$ rotation wise, this indicates an increase in number of balls following the ideal trajectory of high-energy exchange in the normal direction for the HM jar, as expected at the design stage.

For an angular velocity ratio $\omega / \Omega+1=-2.6$, the efficiency of the $C Y$ jar becomes the highest, while $\mathrm{HM}$ exchanges markedly less energy (see Figs. 4 and 5). Beyond this value, a ball-rolling mode establishes in both jars and high-relative-velocity impacts almost vanish. CY jar effi- ciency is still higher than HM's but, as discussed later, experimental data demonstrate that this condition is generally not so effective. The right side of Fig. 5 reports the tangential component of the relative velocities of colliding bodies: for higher-relative-velocity events, CY always exceeds the HM jar.

So far, only the steel-steel case (i.e. no powder charge) has been discussed. To validate model predictions, a comparison of simulations and experimental XRPD data was performed for calcium fluoride (milled at $\Omega=200 \mathrm{rpm}$ for $32 \mathrm{~h}$ ). As already stated, the effect of the mill charge in simulations has been accounted for by modifying the coefficients in the friction parameters ( $\mu$ s and $\mu d$, see Table 2).

For the HM jar, Fig. 7a illustrates the comparison between the impact power density computed for plate velocity $\Omega=200 \mathrm{rpm}$, both for the steel-steel (no feed) and the steel-fluorite interaction cases. As shown for the CY jar in [11], the presence of a fluorite charge reduces friction and (i) shifts the $\omega / \Omega+1$ position of the maximum efficiency to more negative values and (ii) increases the absolute value of the exchanged power density in the $\mathrm{HM}$ jar too. In particular, the broad plateau centered at $\omega / \Omega+1=-1.8$ found for the steel-steel interaction is replaced by a sharp maximum at $\omega / \Omega+1=-2.2$. The same figure also reports results of the WPPM analysis of experimental XRPD patterns collected on fluorite samples ground in the HM jar under the same conditions as those considered in the

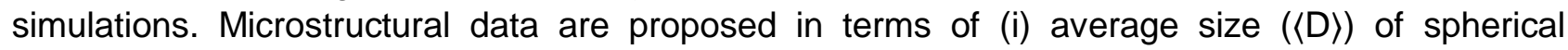
crystallites (i.e. coherent scattering domains) and (ii) dislocation density $(\rho)$. In particular, the more efficient the process, the more $\langle D\rangle$ is expected to decrease (better comminution) whereas $\rho$, which broadly includes a large variety of defects, is supposed to increase [39-45]. These quantities can be 
also related to the mechanical properties of the end product, by means of two expressions describing the effect on microstructure of the milling process; namely, (i) the Hall-Petch relation [46,47],

$$
\sigma_{y}=\sigma_{i}+\frac{k_{y}}{\sqrt{\langle D\rangle}},
$$

where $\sigma y$ is the yield stress, $\sigma i$ the starting stress for dislocations movement and ky the strengthening coefficient of the material; and (ii) the Taylor equation of work hardening [48],

$$
\sigma_{y}=\sigma_{i}+\alpha G b \sqrt{\rho}
$$

where $G$ is the shear modulus, $b$ the magnitude of Burgers vector and $\alpha$ a correction factor specific to the material. From these two relations it can be deduced that, the smaller the grain size, the greater is $\sigma y$ and, the higher is $\rho$, the more hardened becomes the material.

Interestingly, Fig. 7a shows that the impact power density computed from simulations nicely maps the Hall-Petch trend of experimental data, lying in between the steel-steel and steel-fluorite cases, thus validating the simulations and the speculations on $\mathrm{HM}$ jar efficiency. The same applies, to a certain extent, to the dislocation density which however shows a steep increase around $\omega / \Omega+1=-0.6$ and a broad plateau below this value.

A comparison of experimental results of fluorite milled in $\mathrm{HM}$ and $\mathrm{CY}$ jars is shown in Fig. 7b. Two points should be noticed: (i) a smaller crystallite size is obtained in the HM jar than in the CY, whereas (ii) the maximum dislocation density is comparable but more peaked for the $\mathrm{CY}$ than for the $\mathrm{HM}$ one. A possible explanation for the former observation, perfectly compatible with computer simulations, lies in the larger fraction of collisions along impact axis (compression), which are more efficient to comminute the brittle powder charge $[9,10,6]$. On the contrary, the cylindrical jar exhibits a relatively larger amount of dislocations at its best performance, due to the higher energy exchange in the tangential mode, which better supports shear deformation components.

In terms of overall performances, it is worth stressing that the $\mathrm{HM}$ jar exhibits a wider range of optimal conditions than the $\mathrm{CY}$, thus allowing more robust control of the milling process, reflected on the homogeneity of the resulting powder in terms of domain (crystallite) size. Indeed, as observed in [36,37], traditional CY jars mostly produce inhomogeneous powders, with a ground fraction (fine) coexisting with less-tonot ground powder (coarse). This information, for the discussed case study, is revealed in Fig. 8, depicting the distribution of crystallite sizes of calcium fluoride powders ground in the $C Y$ and $H M$ jars at increasing velocity ratios, $\omega / \Omega+1$. XRPD data modelling were obtained by WPPM analysis [31] by imposing two lognormal distributions, corresponding respectively to the fine and the coarse fluorite fractions. The existence of these two fractions was also supported by Scanning Electron Microscopy images (see supplementary information in [12]) and confirmed by solid state Nuclear Magnetic Resonance (NMR) spectroscopy studies [36,37]. As shown in Fig. 8, powder ground inside the HM jar is homogeneous (namely a single lognormal curve is enough to model the data) across a relatively broader range of $\omega / \Omega+1$ velocity ratios than the CY. A more detailed discussion, including the effect of milling time, is reported in [12].

\section{Conclusions}

In this work, an innovative jar design for the planetary ball mill has been proposed. The aim of the design was to enhance comminution of the mill charge by providing an increased fraction of energy exchange along the impact, axis with respect to a traditional cylindrical vial. To pursue this goal, a flat wall portion was inserted halfway between the original curved wall and the axis of the jar, resulting in an half-moon cross-section. A rigorous investigation of the $\mathrm{HM}$ jar and a comparison with the $\mathrm{CY}$ jar has been presented, employing both a dynamic-mechanical multibody model and X-ray powder 
diffraction data. Specifically, the effect of jar and plate angular velocities on vials efficiency has been discussed in terms of impact power density and the probability distribution of relative velocities of colliding bodies, both divided into normal and perpendicular components with respect to the impact axis. Although globally exchanging less energy than the $\mathrm{CY}$ jar, the normal fraction is higher for the $\mathrm{HM}$ vial which also provides a larger amount of high-normal-relative-velocity impacts, supposed to be the most effective in the fracture process of brittle powders (calcium fluoride in this study). Moreover, the range of jar-to-plate velocity ratio, corresponding to the highest efficiency (and most disordered ball motion), is wider for the $\mathrm{HM}(\omega / \Omega+1$ from -1.8 to -2.2$)$ than for the $C Y$ jar (peaked at $\omega / \Omega+1=-2.6$ ). Therefore, being less sensitive to milling parameters, the HM jar provides more robust grinding. Modelling predictions are supported and validated by X-ray Powder Diffraction Line Profile Analysis, giving insights into average crystallite size and defect content of the ground powder. While the CY jar prodeces a larger amount of defects and a homogeneous ground powder only at best milling condition, the HM gives a more uniform and finer end product across a broader range of jar-to-plate velocity ratios. This ongoing research proceeds with the study of different jar geometries, to enhance either the normal or the perpendicular fraction of exchanged energy allowing for the tailoring of the end product characteristics.

\section{Acknowledgements}

The present work was partly supported by Fondazione Caritro, under project No 2013-0247 "Mechanical activation to improve bioavailability and reduce adverse effects of drugs in the therapy of chronic diseases". The authors are especially grateful to Dr. D. Catelani (MSC Software) for precious collaboration and support to the research work. NMP is supported by the European Research Council (ERC StG Ideas 2011 BIHSNAM n. 279985, ERC PoC 2015 SILKENE nr. 693670) and by the European Commission under the Graphene Flagship (WP14 Polymer Composites, no. 696656).

\section{References}

[1] H.J. Fecht, E. Hellstern, Z. Fu, W.L. Johnson, Nanocrystalline metals prepared by highenergy ball milling, Metall. Trans. A 21 (9) (1990) 2333-2337.

[2] VV.AA, High-energy Ball Milling: Mechanochemical Processing of Nanopowders, Woodhead Publishing, 2010.

[3] C. Suryanarayana, Mechanical alloying and milling, Prog. Mater. Sci. 46 (12) (2001) 1-184.

[4] V. Šepelák, S. Bégin-Colin, G. Le Caër, Transformations in oxides induced by high-energy ballmilling, Dalton Trans. 41 (2012) 11927-11948.

[5] S. Indris, D. Bork, P. Heitjans, Nanocrystalline oxide ceramics prepared by highenergy ball milling, J. Mater. Synth. Process. 8 (3-4) (2000) 245-250.

[6] M. Vittori Antisari, A. Montone, N. Jovic, E. Piscopiello, C. Alvani, L. Pilloni, Low energy pure shear milling: a method for the preparation of graphite nano-sheets, Scr. Mater. 55 (11) (2006) 10471050.

[7] K. Kouroupis-Agalou, A. Liscio, E. Treossi, L. Ortolani, V. Morandi, N.M. Pugno, V. Palermo, Fragmentation and exfoliation of 2-dimensional materials: a statistical approach, Nanoscale 6 (2014) 5926-5933.

[8] C.F. Burmeister, A. Kwade, Process engineering with planetary ball mills, Chem. Soc. Rev. 42 (2013) 7660-7667. 
[9] P. Baláz, M. Achimovičová, M. Baláz, P. Billik, Z. Cherkezova-Zheleva, J.M. Criado, F. Delogu, E. Dutková, E. Gaffet, F.J. Gotor, R. Kumar, I. Mitov, T. Rojac, M. Senna, A. Streletskii, K. WieczorekCiurowa, Hallmarks of mechanochemistry: from nanoparticles to technology, Chem. Soc. Rev. 42 (2013) 7571-7637.

[10] S.R. Chauruka, A. Hassanpour, R. Brydson, K.J. Roberts, M. Ghadiri, H. Stitt, Effect of mill type on the size reduction and phase transformation of gamma alumina, Chem. Eng. Sci. 134 (2015) 774-783.

[11] M. Broseghini, L. Gelisio, M. D'Incau, C.L. Azanza Ricardo, N.M. Pugno, P. Scardi, Modeling of the planetary ball-milling process: the case study of ceramic powders, J. Eur. Ceram. Soc. 36 (9) (2016) 2205-2212.

[12] M. Broseghini, M. D'Incau, L. Gelisio, N.M. Pugno, P. Scardi, Homogeneity of ball milled ceramic powders: effect of jar shape and milling conditions, Mater. Des. (2016) (Data in Brief, submitted for publication).

[13] K.D.M. Harris, Mechanochemical synthesis: how grinding evolves, Nat. Chem. 5 (1) (2013) 1214.

[14] T. Frišvcić, I. Halasz, P.J. Beldon, A.M. Belenguer, F. Adams, S.A.J. Kimber, V. Honkimäki, R.E. Dinnebier, Real-time and in situ monitoring of mechanochemical milling reactions, Nat. Chem. 5 (2013) 66-73.

[15] N. Burgio, A. Iasonna, M. Magini, S. Martelli, F. Padella, Mechanical alloying of the fe-zr system. Correlation between input energy and end products, II Nuovo Cimento 13 (4) (1991) 459-476.

[16] Fritsch Vario-Planetary Mill PULVERISETTE 4, http://www.fritsch-milling.com/ products/milling/planetary-mills/pulverisette-4-classic-line/description/.

[17] MSC Adams, http://www.mscsoftware.com/it/product/adams.

[18] H.M. Hilber, T.J.R. Hughes, R.L. Taylor, Improved numerical dissipation for time integration algorithms in structural dynamics, Earthq. Eng. Struct. Dyn. 5 (3) (1977) 283-292.

[19] D. Negrut, R. Rampalli, G. Ottarsson, A. Sajdak, On an implementation of the HilberHughesTaylor method in the context of index 3 differential-algebraic equations of multibody dynamics (detc2005-85096), J. Comput. Nonlinear Dyn. 2 (1) (2006) 73-85.

[20] S. Rosenkranz, S. Breitung-Faes, A. Kwade, Experimental investigations and modelling of the ball motion in planetary ball mills, Powder Technol. 212 (1) (2011) 224-230.

[21] CSM Instruments - Pin-on-Disk Tribometer (TRB), http://www.csm-instruments. com/en/Pin-onDisk-Tribometer.

[22] G. Gilardi, I. Sharf, Literature survey of contact dynamics modelling, Mech. Mach. Theory 37 (10) (2002) 1213-1239.

[23] S. Dubowsky, F. Freudenstein, Dynamic analysis of mechanical systems with clearances - part 1: formation of dynamic model, J. Eng. Ind. 93 (1) (1971) 305-309.

[24] A.H. Kharaz, D.A. Gorham, A.D. Salman, An experimental study of the elastic rebound of spheres, Powder Technol. 120 (3) (2001) 281-291.

[25] W. Goldsmith, Impact: The Theory and Physical Behaviour of Colliding Solids, Dover Publications, 2001. 
[26] J. Kano, F. Saito, Correlation of powder characteristics of talc during planetary ball milling with the impact energy of the balls simulated by the particle element method, Powder Technol. 98 (2) (1998) 166-170.

[27] J. Kano, H. Mio, F. Saito, Correlation of size reduction rate of inorganic materials with impact energy of balls in planetary ball milling, J. Chem. Eng. Jpn 32 (4) (1999) 445-448.

[28] H. Mio, J. Kano, F. Saito, K. Kaneko, Effects of rotational direction and rotation-to-revolution speed ratio in planetary ball milling, Mater. Sci. Eng. A 332 (12) (2002) 75-80.

[29] H. Mio, J. Kano, F. Saito, K. Kaneko, Optimum revolution and rotational directions and their speeds in planetary ball milling, Int. J. Miner. Process. 74 (2004) 85-92 (Special Issue Supplement Comminution 2002).

[30] J.P. Cline, R.D. Deslattes, J.L. Staudenmann, E.G. Kessler, L.T. Hudson, A. Henins, R.W. Cheary, Certificate Srm 660a, NIST, Gaithersburg, MD, USA, 2000.

[31] P. Scardi, Microstructural properties: lattice defects and domain size effects, in: R.E. Dinnebier, S.J.L. Billinge (Eds.), Powder Diffraction. Theory and Practice, RSC Publishing, Cambridge 2008, pp. 378-416 (chapter 13).

[32] M. D'Incau, High Energy Milling in Nanomaterials Technologies: Process Modelling and Optimization(Doctoral Thesis) University of Trento, 2008.

[33] P. Scardi, M. Leoni, Diffraction line profiles from polydisperse crystalline systems, Acta Crystallogr. A 57 (5) (2001) 604-613.

[34] P. Scardi, M. Leoni, Whole powder pattern modelling, Acta Crystallogr. A 58 (2) (2002) 190200.

[35] G. De Giudici, R. Biddau, M. Dincau, M. Leoni, P. Scardi, Dissolution of nanocrystalline fluorite powders: an investigation by $\{\mathrm{XRD}\}$ and solution chemistry, Geochim. Cosmochim. Acta 69 (16) (2005) 4073-4083.

[36] M. Abdellatief, M. Abele, M. Leoni, P. Scardi, Solid state nuclear magnetic resonance and X-ray diffraction line profile analysis of heavily deformed fluorite, Thin Solid Films 530 (2013) 44-48.

[37] M. Abdellatief, M. Abele, M. Leoni, P. Scardi, Combined x-ray diffraction and solidstate $19 f$ magic angle spinning $\mathrm{nmr}$ analysis of lattice defects in nanocrystalline caf2, J. Appl. Crystallogr. 46 (4) (2013) 1049-1057.

[38] S. Sadrai, J.A. Meech, D. Tromans, F. Sassani, Energy efficient comminution under high velocity impact fragmentation, Miner. Eng. 24 (10) (2011) 1053-1061.

[39] E. Hellstern, H.J. Fecht, Z. Fu, W.L. Johnson, Structural and thermodynamic properties of heavily mechanically deformed ru and alru, J. Appl. Phys. 65 (1) (1989) 305-310.

[40] J.Y. Huang, Y.K. Wu, H.Q. Ye, Ball milling of ductile metals, Mater. Sci. Eng. A 199 (2) (1995) $165-172$.

[41] Á. Révész, T. Ungár, A. Borbély, J. Lendvai, Dislocations and grain size in ball-milled iron powder, Nanostruct. Mater. 7 (7) (1996) 779-788.

[42] L. Börner, J. Eckert, Nanostructure formation and steady-state grain size of ballmilled iron powders, Mater. Sci. Eng. A 226 (1997) 541-545 (Ninth International Conference on Rapidly Quenched and Metastable Materials).

[43] S. Vives, E. Gaffet, C. Meunier, X-ray diffraction line profile analysis of iron ball milled powders, Mater. Sci. Eng. A 366 (2) (2004) 229-238. 
[44] O. Boytsov, A.I. Ustinov, E. Gaffet, F. Bernard, Correlation between milling parameters and microstructure characteristics of nanocrystalline copper powder prepared via a high energy planetary ball mill, J. Alloys Compd. 432 (2007) 103-110.

[45] M. D'Incau, M. Leoni, P. Scardi, High-energy grinding of femo powders, J. Mater. Res. 22 (2007) 1744-1753.

[46] E.O. Hall, The deformation and ageing of mild steel: III discussion of results, Proc. Phys. Soc. London, Sect. B 64 (9) (1951) 747-753.

[47] N.J. Petch, The cleavage strength of polycrystals, J. Iron Steel Inst. 174 (1953) 25-28.

[48] G.I. Taylor, The mechanism of plastic deformation of crystals. Part I. Theoretical, Proc. R. Soc. Lond. A 145 (855) (1934) 362-387.

Table 1

Physical properties of CY, HM jars and milling media for the presented case study.

\begin{tabular}{llll}
\hline CY, HM jars (AISI 304) & \multicolumn{3}{l}{ Spheres (AISI C1020) } \\
\hline Density & $8.03 \mathrm{~g} / \mathrm{cm}^{3}$ & Density & $7.85 \mathrm{~g} / \mathrm{cm}^{3}$ \\
Young modulus & $193 \mathrm{GPa}$ & Young modulus & $200 \mathrm{GPa}$ \\
Poisson ratio & 0.29 & Poisson ratio & 0.29 \\
\hline
\end{tabular}

Table 2

Values introduced in the contact and friction models and methods used for their derivation.

\begin{tabular}{lll} 
Sphere-sphere generalized contact stiffness, $k_{s s}$ & Hertz theory \\
Sphere-plane generalized contact stiffness, $k_{s p}$ & $252,146 \mathrm{~N} / \mathrm{mm}^{3 / 2}$ & Hertz theory \\
Restitution coefficient, $e$ & $350,236 \mathrm{~N} / \mathrm{mm}^{3 / 2}$ & Drop test, $e=\sqrt{h / h_{0}}$ \\
Sphere-sphere contact damping, $c_{m, s s}$ & 0.516 & Damped mass-spring system solution \\
Sphere-plane contact damping, $c_{m, s p}$ & $10.1 \mathrm{~kg} / \mathrm{s}$ & Damped mass-spring system solution \\
Steel-Steel $\mu_{s}$ & $17.5 \mathrm{~kg} / \mathrm{s}$ & Literature \\
Steel-Steel $\mu_{d}$ & 1.00 & Tribological test \\
Steel-Fluorite $\mu_{s}$ & 0.80 & Literature \\
Steel-Fluorite $\mu_{d}$ & 0.25 & Tribological test \\
\hline
\end{tabular}



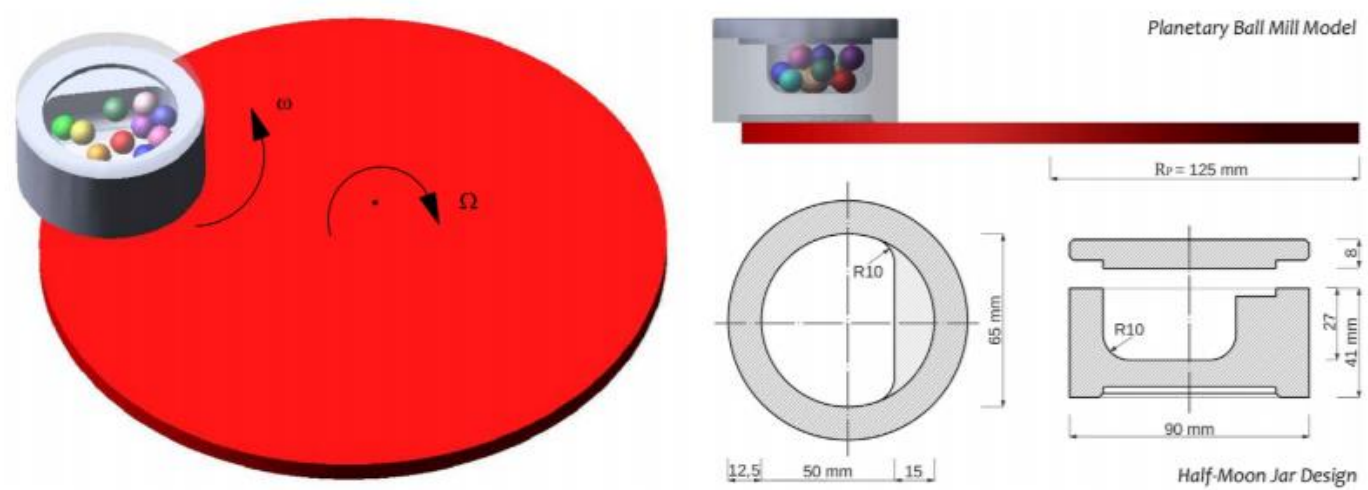

Half-Moon Jar Design

Fig. 1. Planetary ball mill installing the half moon jar and definition of rotation $(\omega)$ and revolution $(\Omega)$ angular velocities. The design of the new half-moon shaped jar is also reported.
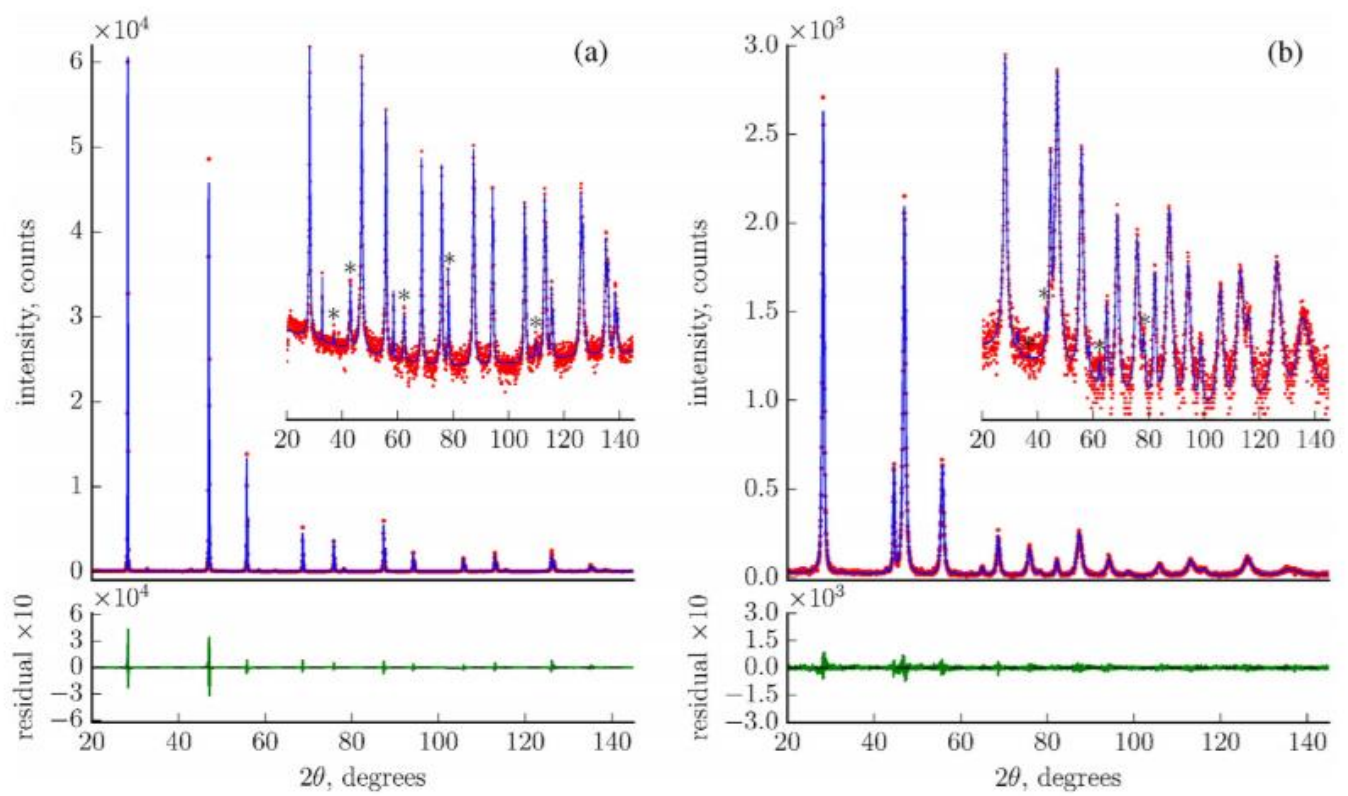

Fig. 2. XRPD pattern of (coarse-grained) pristine fluorite powder (a); same powder after ball-milling in half-moon jar, for $32 \mathrm{~h}$ at $\omega / \Omega+1=-2.2$ (b). Experimental data (red circle) are shown with the WPPM profile (blue line), and their difference (residual, green line below). The inset shows the same data in log scale to highlight details and profile modelling quality: some of the weak peaks ( $\left(^{*}\right.$ are from MgO, present as impurity phase and also included in the WPPM. (For interpretation of the references to color in this figure legend, the reader is referred to the web version of this article.) 
Cylindrical Jar

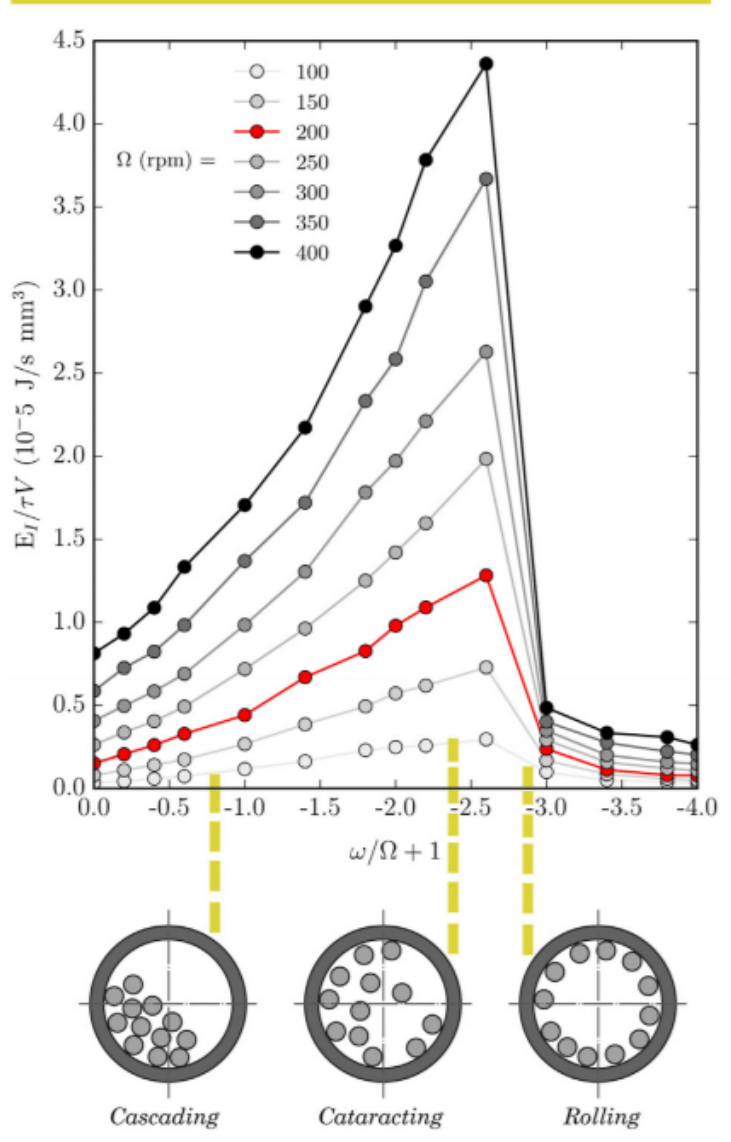

Half-Moon Jar

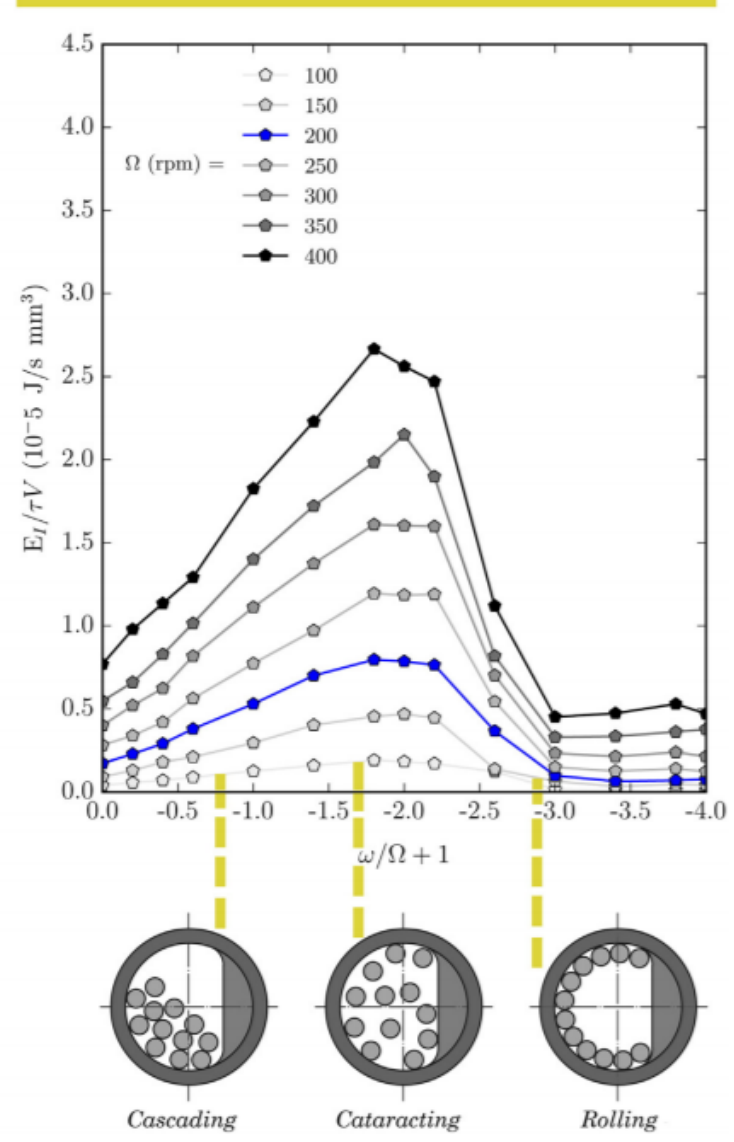

Fig. 3. Impact power density (Eq. (2)) for the steel-steel interaction, for the cylindrical (left) and the half moon (right) jars. Curves are computed for different plate velocities ( $\Omega$ ). The typical ball trajectories corresponding to the different $\omega / \Omega+1$ velocity ratios are also sketched. 


\section{Cylindrical Jar}

(a) $\Omega=200 \mathrm{rpm}$, balls contacts contribution

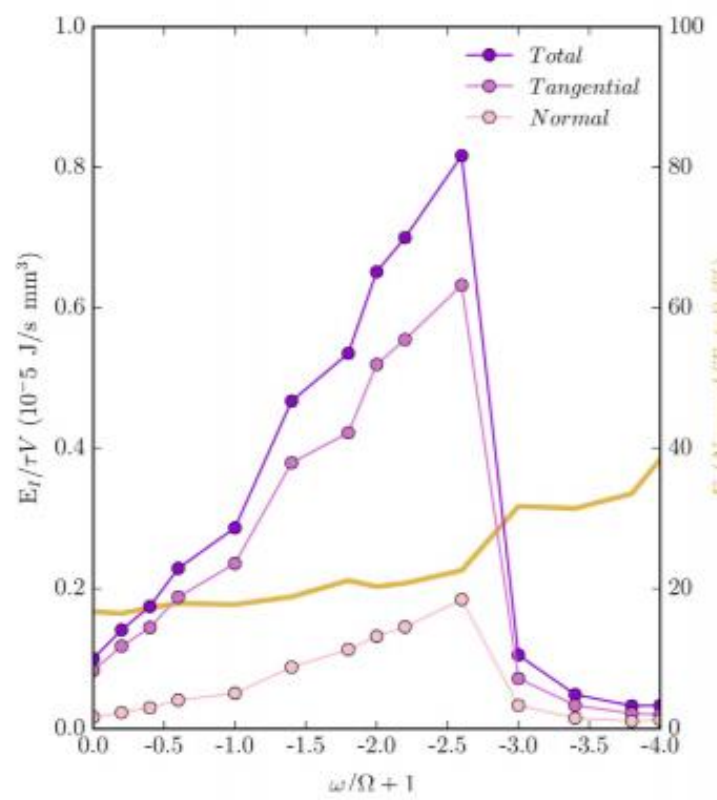

(b) $\Omega=200 \mathrm{rpm}$, balls -jar contacts contribution

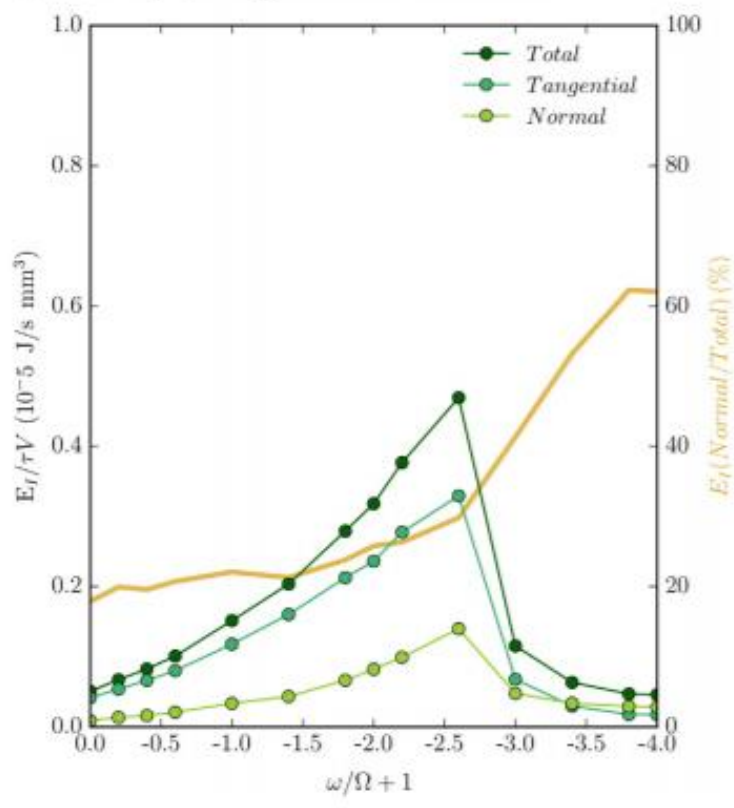

Half-Moon Jar
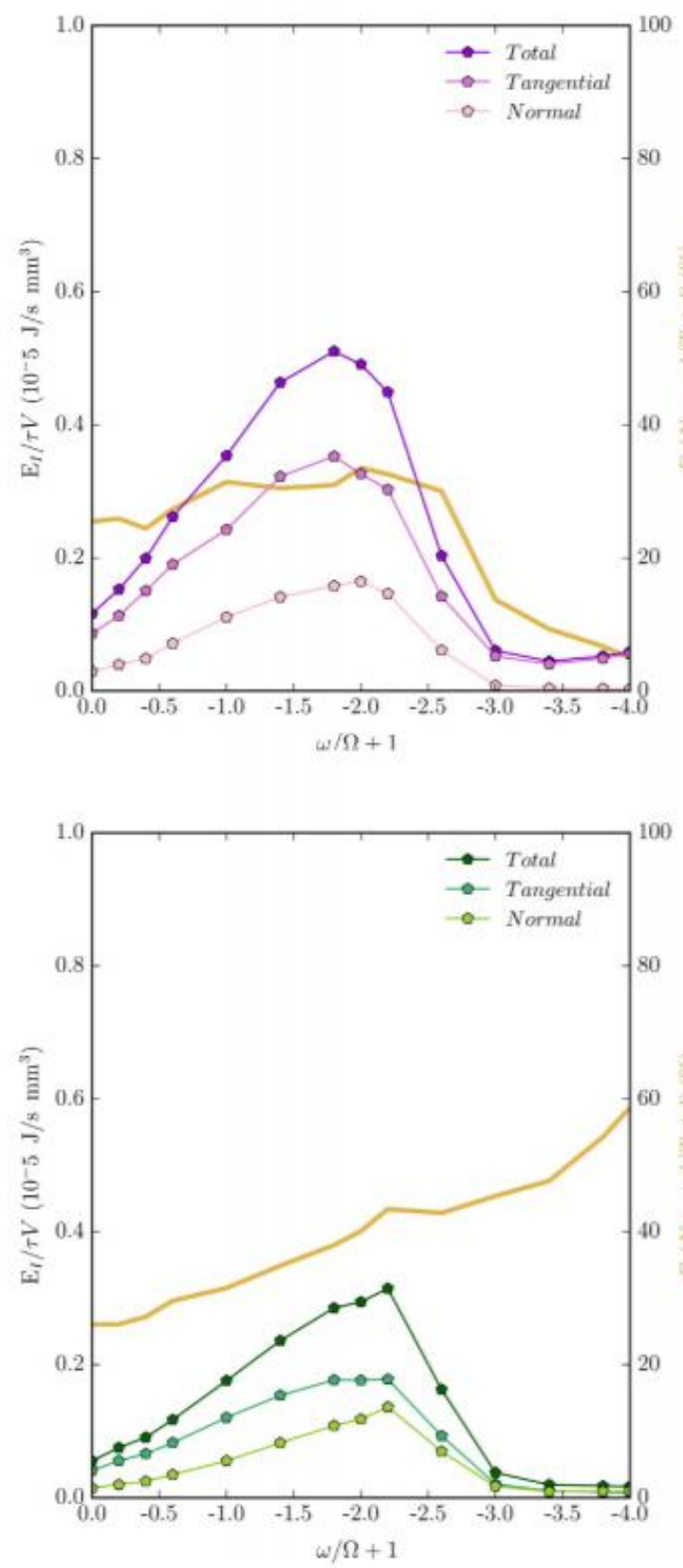

Fig 4. Impact power density (Eq. (2)) for the steel-steel interaction, for the cylindrical (left) and the half moon (right) jar computed for $\mathrm{\Omega}=200$ rpm and divided into ball-ball and balls-jar contacts contributions. These quantities are also broken down into normal and tangential components (with respect to the local impact reference frame). The yellow line depicts the fraction of energy exchanged along the impact axis ( $E_{l}($ normal/total)). (For interpretation of the references to color in this figure legend, the reader is referred to the web version of this article.) 

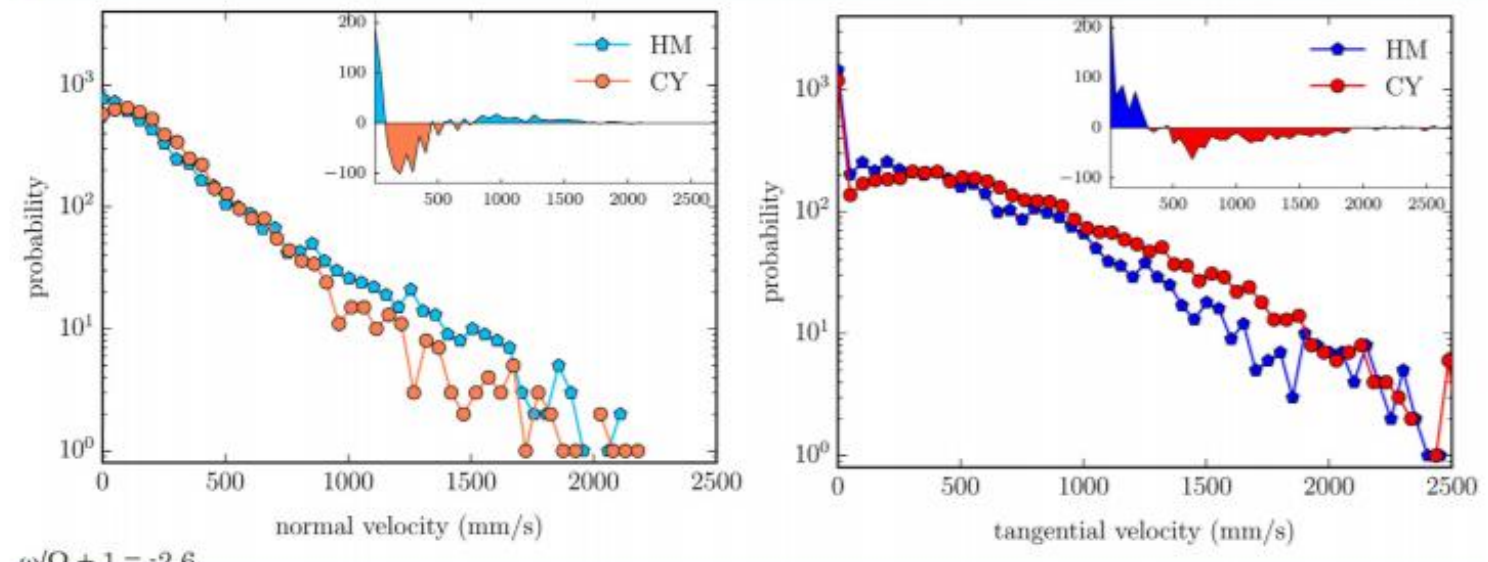

$\omega / \Omega+1=-2.6$
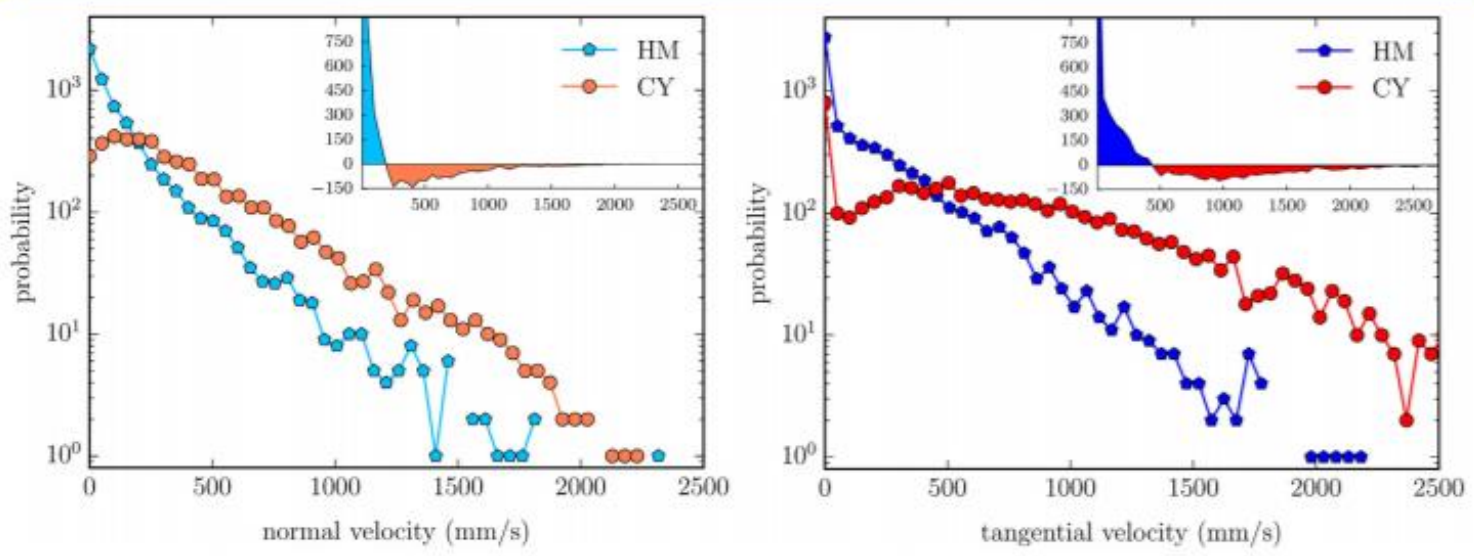

Fig. 5. Probability distribution of normal (penetration, left) and tangential (slip, right) components of the relative velocity of colliding bodies for both $\omega / \Omega+1=-1.8$ and -2.6 (being $\Omega=200 \mathrm{rpm}$ ), respectively corresponding to the highest-efficiency condition for the $\mathrm{HM}$ and $\mathrm{CY}$ jars. The insets report, in linear scale, the difference between HM and $\mathrm{CY}$ probability distributions.
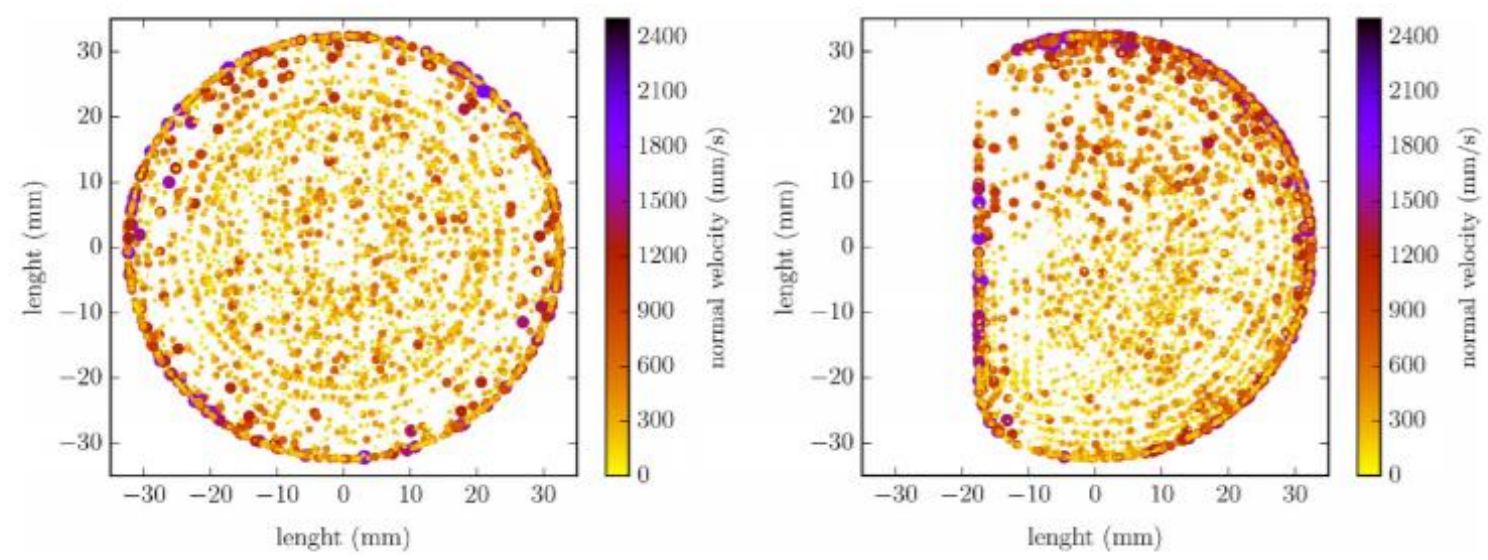

Fig. 6. Map of balls-jar contact events in the $\mathrm{CY}$ (left) and $\mathrm{HM}$ (right) jars at $\Omega=200 \mathrm{rpm}$ and $\omega / \Omega+1=-1.8$, for the steel-steel interaction case. The relative velocities during each collision define the color scale and the radius of each point. HM jar ciearly shows a concentration of events in the upper comer. 

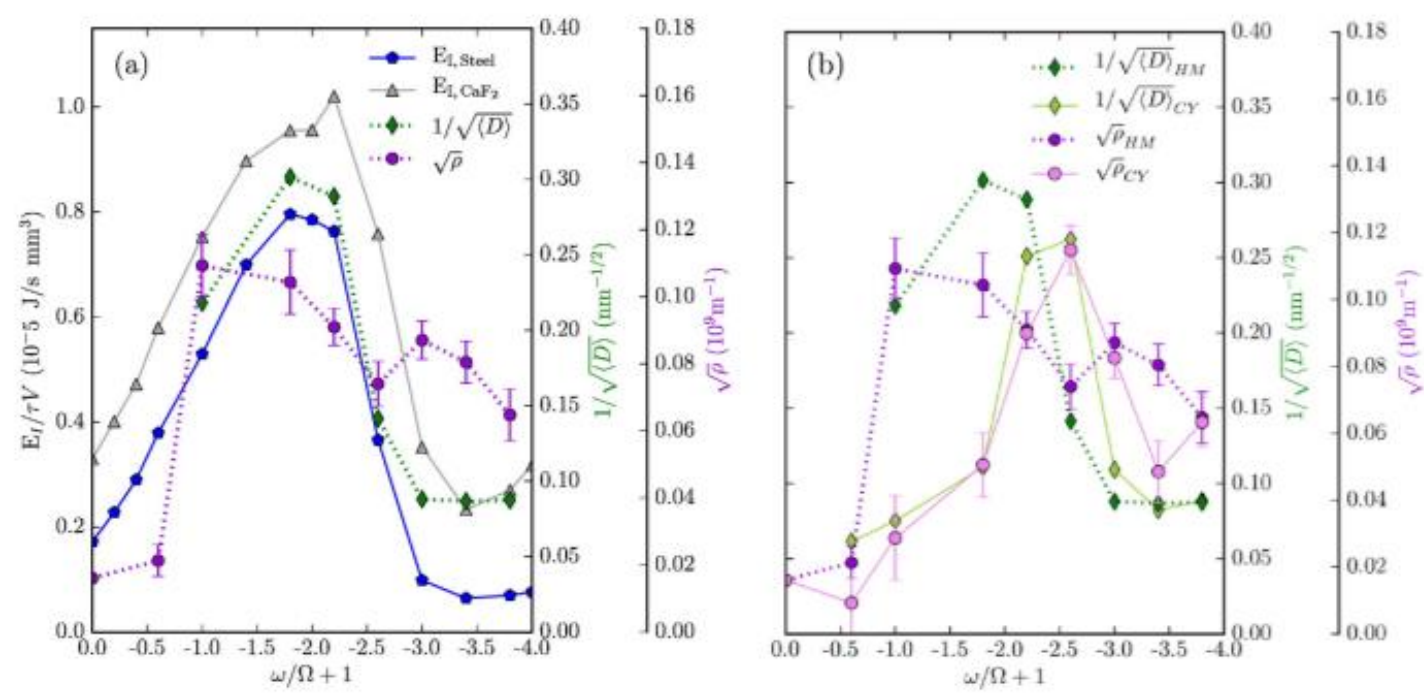

Fig. 7. Left (a), comparison of simulated and experimental data collected for calcium fluoride ground in the HM jar. Impact power density computed from simulations for the steel-steel ( $E_{l, S m o s}$ blue pentagons) and the steel-fluorite ( $E_{l, C a f}$, grey triangles) interactions nicely maps the trend of experimental data expressed as the reciprocal of the square root of the average crystallite size $(1 / \sqrt{\langle\bar{D})}$, green diamonds), calculated from the X-ray powder diffraction pattern of milled calcium fluoride. The dislocation density ( $\sqrt{\rho}$, violet circles) is also reported, expressing the deviation from the corresponding ideal structure. Right (b), the same experimental data for the $\mathrm{CY}$ and $\mathrm{HM}$ jars are compared, illustrating the difference between the two jar shapes, in terms of crystallite size and dislocation density, characterizing the end-product at each velocity ratio. Interestingly, while the maximum of these two quantities coincides for the $\mathrm{CY}$ case, it is different for the $\mathrm{HM}$ jar. Both for experimental and computed data, $\Omega=200 \mathrm{rpm}$. (For interpretation of the references to color in this figure legend, the reader is referred to the web version of this article.
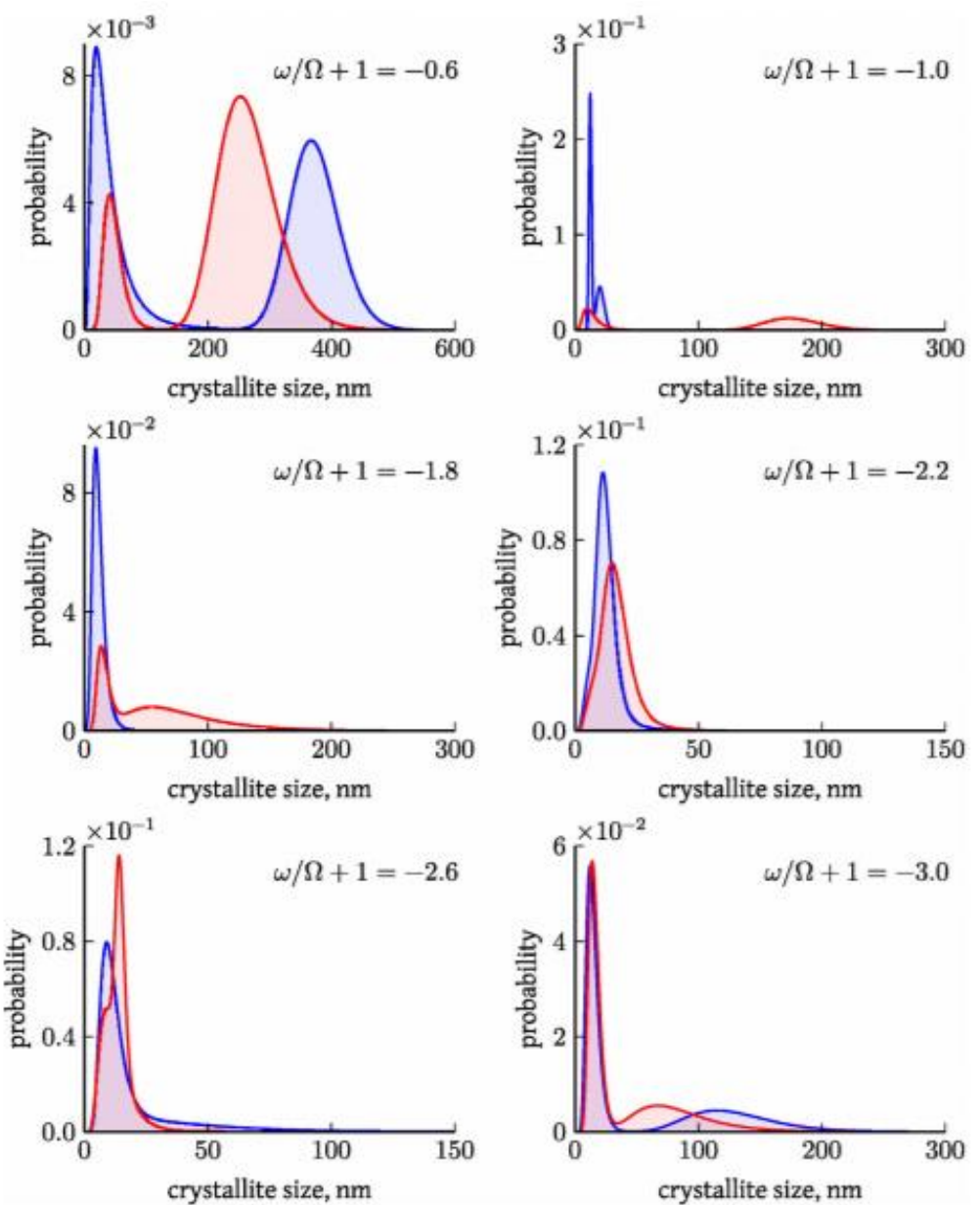

Fig. 8. WPPM models of crystalline domain size probability distribution of fluorite ground in $\mathrm{CY}$ (red) and $\mathrm{HM}$ (blue) jars for $32 \mathrm{~h}$ at increasing velocity ratios, $\omega / \Omega+1$. $\mathrm{HM}$ jar produces an homogeneous size distribution powder (one lognormal curve) for a wider range of $\omega / \Omega+1$ than $C Y$. (For interpretation of the references to color in this figure legend, the reader is referred to the web version of this article.) 